\title{
Correction to: The Tannaka-Krein Formalism and (Re)Presentations of Universal Quantum Groups
}

\author{
Theo Raedschelders and Michel Van den Bergh
}

\section{Correction to:}

Chapter 15 in: Y. I. Manin, Quantum Groups

and Noncommutative Geometry, CRM Short Courses, https://doi.org/10.1007/978-3-319-97987-8_15

The original version of the book was inadvertently published with book author name "Yuri I. Manin" in chapter "The Tannaka-Krein Formalism and (Re) Presentations of Universal Quantum Groups", which has been now rectified and changed to "Theo Raedschelders and Michel Van den Bergh" in the online version of the chapter and book, and also in the printed book. 